Arq. Bras. Med. Vet. Zootec., v.68, n.6, p.1479-1486, 2016

\title{
Perfil hematológico de éguas Quarto de Milha alimentadas com feno ou haylagede Tifton-85 (Cynodon spp.)
}

\author{
[Hematological panel of Quarter Horse mares fed Tifton 85 (Cynodon spp.) hay and haylage] \\ T.C. Guimarães ${ }^{1}$, A.S.C. Rezende ${ }^{2}$, M.L.L. Costa ${ }^{1}$, M.G. Ferreira ${ }^{1}$, \\ B. Soto-Blanco ${ }^{1}$, M.M. Melo ${ }^{1 *}$ \\ ${ }^{1}$ Universidade Federal de Minas Gerais - Belo Horizonte, MG \\ ${ }^{2}$ Universidade Federal da Paraíba - Santa Rita, PB
}

\begin{abstract}
RESUMO
O capim Tifton-85 (Cynodon spp.) vem sendo utilizado na alimentação de equinos na forma de présecado (haylage) e feno, todavia não existem estudos conclusivos a respeito da influência da haylage sobre o estado de saúde dessa espécie. Portanto, esta pesquisa objetivou avaliar o perfil sanguíneo como indicador de higidez de éguas Quarto de Milha alimentadas com haylage e feno de Tifton-85. A gramínea utilizada foi cortada com 30 dias de crescimento. Para produção da haylage, a planta cortada permaneceu no campo até atingir $70 \%$ de matéria seca, quando foi colhida. Foram utilizadas 12 éguas Quarto de Milha, com idade entre oito e 12 anos e com peso vivo médio inicial de 451,6kg. Os animais foram distribuídos aleatoriamente em dois grupos $(\mathrm{n}=6)$, sendo o grupo 1 alimentado com feno e o grupo 2 alimentado com haylage, por um período de 28 dias. Foram realizadas cinco coletas de sangue em intervalos de uma semana, para a determinação do perfil hematológico. Em relação ao eritrograma, observou-se discreta diminuição da concentração de hemoglobina, do hematócrito e do volume globular médio (VGM) em ambos os grupos. No leucograma, houve aumento dos leucócitos totais nas coletas iniciais em ambos os grupos, mas sem significado clínico. Conclui-se que éguas adultas Quarto de Milha, após a ingestão de feno e haylage de Tifton-85, não apresentaram alterações no perfil hematológico que indicassem prejuízos à saúde.
\end{abstract}

Palavras-chave: Tifton, haylage, conservação de alimentos, hemograma, equinos

\begin{abstract}
Tifton-85 grass (Cynodon spp) has been used in equine nutrition in the form of pre-dried (haylage) and hay, however, there are no conclusive studies about the influence of haylage on blood profile of this species. Therefore, the aim of this study was to evaluate the blood profile as health indicators of Quarter Horse mares were fed haylage and Tifton-85 hay. The used grass was cut at 30 days of growth. For the production of haylage, this plant remained cut in the field until it had $70 \%$ of dry matter, when it was collected. Twelve Quarter Horse mares, aged 8-12 years, with average weight of 451.58kg were used. The animals were distributed randomly into two groups $(n=6)$, group 1 fed hay and group 2 fed haylage, both for 28 days. Blood samples were collected at one week intervals each for determining the hematological parameters. In the erythrogram, a decrease in the hemoglobin concentration, packed cell volume and mean cell volume after the administration in both groups were detected. The white blood count increased in both groups, but the values remained within normal limits without clinical significance. In conclusion, adult Quarter Horse mares fed Tifton-85 hay and haylage showed no alterations in hematology.
\end{abstract}

Keywords: Tifton, haylage, food conservation, hemogram, horses

Recebido em 7 de setembro de 2015

Aceito em 17 de junho de 2016

*Autor para correspondência (corresponding author)

E-mail: mariliamm@ufmg.br 


\section{INTRODUÇÃO}

Segundo dados de 2010, o Brasil possuía 5.496.817 equinos, sendo Minas Gerais o estado que apresenta o maior percentual do rebanho nacional (14,56\%), com cerca de 800.108 animais. Nesse estado, o maior número de equídeos não tem raça definida $(37,13 \%)$, e, entre as raças criadas com objetivo comercial, a Mangalarga Marchador é a mais numerosa $(33,66 \%)$, seguida pelas raças Campolina $(12,21 \%)$ e Quarto de Milha (4,13\%) (Vieira et al., 2015).

Para garantir a oferta de volumosos para alimentação desse rebanho de equinos, é necessário que parte das forragens seja conservada. As formas de conservação das forragens mais utilizadas na alimentação animal são os fenos e as silagens, ainda que essa última seja utilizada em menor escala na alimentação de equinos, pois, algumas vezes, não apresenta boa palatabilidade por ser rapidamente fermentável, principalmente nas regiões onde prevalecem altas temperaturas e umidade do ar, podendo estar associada à presença de fungos e seus metabólitos. No entanto, as silagens podem favorecer o desenvolvimento de bactérias do gênero Clostridium, o que possibilita a presença de suas toxinas nos silos (Domingues, 2009).

Haylage pode ser definida como forragem emurchecida e ensilada, com alto teor de matéria seca, que atinge níveis acima de $50 \%$, podendo atingir $75 \%$, sendo confeccionada no formato de pequenos fardos, com aproximadamente $20 \mathrm{~kg}$. O mecanismo de conservação é semelhante ao das demais silagens, contudo o processo fermentativo é limitado, o que consequentemente reduz a produção de ácidos orgânicos e a queda do $\mathrm{pH}$, sendo, por isso, mais indicada para compor a dieta dos equinos. A haylage é amplamente utilizada em diversos países da Europa, inclusive em substituição ao feno (Müller e Udén, 2007; Ragnarsson e Lindberg, 2008). Entretanto, no Brasil, alguns proprietários atribuem prejuízos à saúde dos equinos após a ingestão da haylage. Uma das alegações seria o baixo valor nutricional deste volumoso. Costa (2012) verificou que a utilização da haylage de Tifton 85 (Cynodon spp.) na dieta de equinos supre adequadamente as necessidades nutricionais dos animais, desmistificando esta afirmativa. Contudo, ainda não foram feitos estudos sobre a influência da haylage sobre a homeostase de equinos. Desse modo, este estudo objetivou determinar os possíveis efeitos da haylage de Tifton 85 sobre o perfil sanguíneo de equinos hígidos.

\section{MATERIAL E MÉTODOS}

O projeto deste estudo foi aprovado pelo Comitê de Experimentação Animal da Universidade Federal de Minas Gerais-UFMG (protocolo 055/10).

A gramínea Tifton-85 (Cynodon spp.) foi cortada com 30 dias de crescimento (28cm de altura) para produção da haylage e permaneceu no campo até atingir $70 \%$ de matéria seca (MS), quando foi recolhida. A MS foi determinada por secagem em forno micro-ondas, segundo técnica descrita por Pastorini et al. (2002). Dessa forma, para quantificação da MS, foi realizada amostragem representativa de vários pontos coletados aleatoriamente no campo, seguidos da formação de um pool para secagem em forno micro-ondas.

O aditivo biológico Silobac ${ }^{\circledR}$ foi adicionado seguindo recomendações do fabricante $(2 \mathrm{~g}$ do produto em $2 \mathrm{~L}$ de água para inocular cada tonelada de forragem destinada à ensilagem). $\mathrm{O}$ produto forneceu, por grama de forragem ensilada, Lactobacillus plantarum e Pediococcus pentosaceus nas concentrações de 2,5 x 10 ${ }^{5} \mathrm{UFC}$ para cada espécie de bactéria. Aproximadamente $1,3 \mathrm{~kg}$ de massa verde da forragem foi acondicionado em sacos de polietileno (40 x $60 \mathrm{~cm}$ ) para produção de haylage. Em seguida, os sacos foram selados após retirada do ar com máquina de vácuo, segundo técnica preconizada por Kung Jr. et al. (2010). As haylages produzidas ficaram armazenadas durante 56 dias, até o momento da abertura para fornecimento aos animais.

Os fardos de fenos foram produzidos com auxílio de maquinário de escala industrial. Os fenos foram estocados sobre estrados de madeira em galpão com ventilação. $\mathrm{O}$ concentrado foi formulado e produzido na própria fazenda. Água e sal mineral (COEQUI Plus - Tortuga Cia. Zootécnica Agrária) foram oferecidos à vontade, em cochos separados. 
A composição química do feno e da haylage utilizados nesse experimento foi, respectivamente: matéria seca (\%) 94,54 e 94,71; matéria orgânica - 91,20 e 90,85; matéria mineral - 8,80 e 9,15; proteína bruta - 11,44 e 12,71; fibra em detergente neutro - 55,16 e 54,77; fibra em detergente ácido - 20,41 e 22,48; hemiceluloses 34,74 e 32,29 ; energia bruta (Mcal/kg) - 4,72 e 4,89 .

Foram utilizadas 12 éguas da raça Quarto de Milha, com idade variando entre oito e 12 anos e peso vivo médio de $451,6 \mathrm{~kg}$ no início do experimento. Uma semana antes do início do experimento, as éguas foram vermifugadas e banhadas com carrapaticida. Os animais foram distribuídos aleatoriamente em dois grupos $(n=6)$, que consistiram os tratamentos experimentais, os quais diferiram pela oferta de volumoso, feno (gru 1) ou haylage (gru 2).

Durante o período experimental, os animais foram mantidos em baias individuais com dimensões de $9 \mathrm{~m}^{2}$. As baias foram forradas com cama de palha de arroz, trocada semanalmente, e foram mantidas limpas. A limpeza dos cochos de água das baias foi feita duas vezes ao dia, de manhã e à tarde. Diariamente, durante uma hora, os animais foram soltos em curral com piso cimentado, onde não tiveram acesso a nenhum tipo de alimento.

Semanalmente, os animais foram pesados para cálculo da quantidade do fornecimento de volumoso e concentrado, sendo também feita a coleta do sangue. $\mathrm{O}$ fornecimento dos nutrientes da dieta foi calculado de acordo com indicação para animais em mantença (Nutrient..., 2007). A quantidade de volumoso calculada foi fracionada em três refeições diárias, nos horários de sete, 13 e 17 horas; enquanto o concentrado diário foi fornecido em duas refeições, às nove e às 15 horas.

As coletas de sangue foram realizadas semanalmente, durante 28 dias, antes do fornecimento diário das dietas, totalizando cinco coletas por animal. Foram coletados $5 \mathrm{~mL}$ de sangue, por meio de punção da veia jugular com agulha para coleta a vácuo, acondicionados em tubos a vácuo com EDTA a $10 \%$ para a realização do hemograma (contagem de eritrócitos, leucócitos e dosagem de hemoglobina) em contador eletrônico Celm®.
Volume globular (VG) foi determinado pela técnica do micro-hematócrito (Jain, 1993). Os esfregaços sanguíneos foram confeccionados após a coleta, corados com panótico, para contagem diferencial de leucócitos, e os índices hematimétricos calculados de acordo com Thrall et al. (2007).

A primeira coleta de sangue foi realizada no tempo zero (T0), e as coletas subsequentes foram obtidas a cada sete dias após a administração de haylage: sete (T1), 14 (T2), 21 (T3) e 28 (T4), totalizando cinco coletas por animal.

O delineamento experimental foi totalmente ao acaso, conforme preconizado por Sampaio (2007), em arranjo de parcelas subdivididas. Bastonetes, basófilos e monócitos foram analisados por testes não paramétricos, MannWhitney para comparar os grupos e Friedman para comparar os momentos das coletas, com nível de significância de $\mathrm{P}<0,05$. As demais variáveis foram analisadas pelo teste $t$ de Student ou pela análise de variância ANOVA, seguidos pelo teste de Tukey, com nível de significância de $\mathrm{P}<0,05$. As variáveis leucócitos, linfócitos, neutrófilos, eosinófilos, monócitos, eritrócitos e CHGM foram transformadas por $\log 10(\mathrm{x}+1)$ para normalização.

\section{RESULTADOS E DISCUSSÃO}

Neste estudo, não foi verificada alteração $(\mathrm{P}<$ 0,05) no número de eritrócitos entre os animais do grupo 1 (feno) com os do grupo 2 (haylage) e entre tempos estudados (Tab. 1). Esses valores encontrados nesta pesquisa estão dentro dos limites de normalidade para em equinos (Clinical..., 1988), exceto, em ambos os grupos, em T3 e T4, mas sem prejuízos à saúde dos animais e sem significado clínico.

As concentrações de hemoglobina (Tab. 1) nos animais do grupo 1 apresentaram redução $(\mathrm{P}<$ 0,05) no T1 (sete dias após a administração do feno) da concentração de hemoglobina, inclusive estando discretamente abaixo do limite mínimo de referência estabelecido (Clinical..., 1988). Apesar de o número de eritrócitos estar dentro dos limites de referência para a espécie, pode-se considerar que ocorreu uma discreta anemia pois, classicamente, a hemoglobina é o primeiro parâmetro que diminui. 
Tabela 1. Valores médios e coeficiente de variação do número de hemácias, concentração de hemoglobina e volume globular (VG) de éguas alimentadas com feno (G1) ou haylage de Tifton-85 (G2)

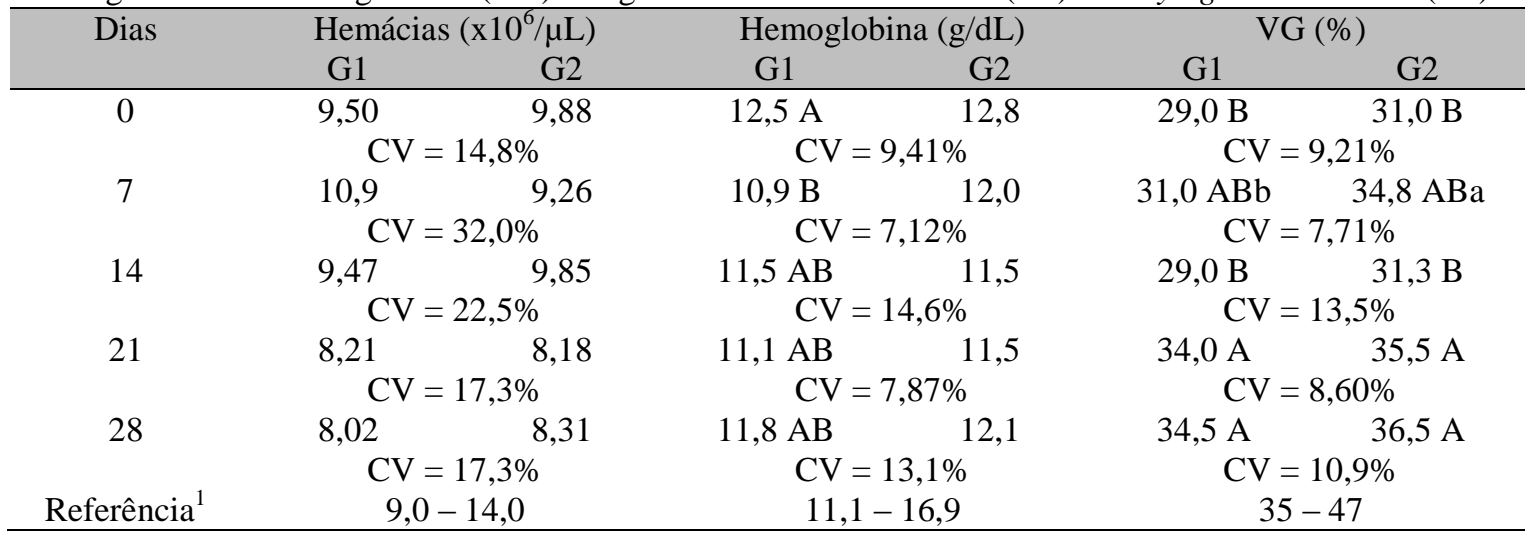

AB: médias seguidas das letras maiúsculas distintas apresentam diferença significante $(\mathrm{P}<0,05$, teste Tukey) entre os tempos de coleta (coluna).

ab: médias seguidas das letras maiúsculas distintas apresentam diferença significante $(\mathrm{P}<0,05$, teste $\mathrm{t}$ de Student) entre os grupos (linhas).

${ }^{1}$ Segundo Clinical..., (1988).

$\mathrm{Na}$ anemia ferropriva, o primeiro estágio é caracterizado por depleção dos estoques de ferro metabolicamente inativos. Tendo em vista que a ferritina e a hemossiderina são o maior compartimento de armazenamento (fígado, medula, baço e outros), a dosagem da ferritina sérica reflete os estoques teciduais desse componente. Este estágio é chamado de depleção de estoque. No segundo estágio, ocorre aumento da capacidade de ligação do ferro e diminuição do ferro sérico, juntamente com diminuição do índice de saturação da ferritina, constituindo uma eritropoiese deficiente. $\mathrm{Na}$ persistência do balanço negativo de ferro, ocorre interferência no processo de hemoglobinização das hemácias, levando a uma diminuição da dosagem de hemoglobina e, consequentemente, do VCM. Este é o terceiro estágio, chamado de anemia ferropriva (Thrall et al., 2007).

No presente estudo, é provável que esta anemia tenha sido um efeito transitório de adaptação à haylage administrada como alimento, mas que foi rapidamente revertida. No entanto, não se pode excluir a possibilidade de esta anemia ser decorrente de uma possível reação fisiológica à infecção por endoparasitas intestinais, apesar dos vermífugos administrados aos animais antes do início do experimento. De fato, Riet-Correia $e t$ al. (2001) e Fortes (2004) relataram que alterações hematológicas, como anemia e leucocitose associadas ou não à eosinofilia, têm sido vistas como consequência do parasitismo intestinal em equinos. Alguns animais podem apresentar infestações parasitárias de forma assintomática, pois sempre haverá algum grau de infestação por parasitas, ressaltando que não necessariamente tal fato possa resultar em alterações clínicas ou prejuízo à saúde animal. Isso dependerá muito do grau de infestação e de resistência do animal à infestação e ao tipo de endoparasita.

Os valores do VG apresentaram-se discretamente abaixo do limite mínimo de referência para a espécie (Clinical..., 1988) em todos os tempos nas éguas do grupo 1 (feno), e nos tempos T0, T1 e T2 nas éguas do grupo 2 (haylage). O VG representa a porcentagem do sangue total composta por eritrócitos. Existem situações em que os valores podem estar diminuídos, especialmente quando ocorre perda sanguínea ou espoliação por endo e ectoparasitos (Thrall et al., 2007). Apesar de os animais terem sido desverminados e banhados com soluções carrapaticidas, estes podem estar apresentando um quadro de recuperação por infestação por endoparasitas, uma vez que animais estabulados tendem a possuir uma reinfestação de endoparasitas mais frequente (Riet-Correa et al., 2001). Como forma de reequilíbrio do organismo, tanto nos grupos feno como nos haylage, na terceira e quarta semanas, ocorreu aumento do VG, indicando uma influência da boa nutrição sobre essa variável sanguínea. 
As anemias, mesmo que discretas, são caracterizadas de acordo com os índices hematimétricos volume globular médio (VGM), concentração de hemoglobina globular média (CHGM) e hemoglobina globular média (HGM). De forma semelhante ao VG, no grupo 1 (feno), nos três primeiros tempos (T0, T1 e T2), os valores do VGM (Tab. 2) apresentaram-se discretamente abaixo do limite mínimo estabelecido para a espécie. Em relação ao grupo 2 (haylage), somente nos tempos T0 e T2 estes valores estão discretamente abaixo. O VGM é o índice que indica o tamanho dos eritrócitos, que, no diagnóstico das anemias, quando reduzido, é denominado microcítico, aumentado é macrocítico, e inalterado, normocítico (Jain, 1993; Thrall et al., 2007). Desse modo, as éguas do grupo 1 (feno), já na primeira coleta, apresentaram discreta anemia do tipo normocítica hipocrômica. No entanto, foi verificado aumento do VGM, após fornecimento da dieta, nos tempos T3 e T4 de ambos os grupos estudados, demonstrando uma boa composição nutricional da forrageira.

Tabela 2. Valores médios e coeficiente de variação de volume globular médio (VGM), concentração de hemoglobulina globular média (CHGM) e hemoglobulina globular média (HGM) de éguas alimentadas com feno (G1) ou haylage de Tifton-85 (G2)

\begin{tabular}{|c|c|c|c|c|c|c|}
\hline \multirow[t]{2}{*}{ Dias } & \multicolumn{2}{|c|}{$\operatorname{VGM}\left(\mu^{3}\right)$} & \multicolumn{2}{|c|}{ CHGM (\%) } & \multicolumn{2}{|c|}{ HGM (pg) } \\
\hline & G1 & G2 & G1 & $\mathrm{G} 2$ & G1 & $\mathrm{G} 2$ \\
\hline \multirow[t]{2}{*}{0} & $30,7 \mathrm{Ba}$ & $32,4 \mathrm{Ba}$ & $43,3 \mathrm{Aa}$ & $41,5 \mathrm{Aa}$ & $13,2 \mathrm{AB}$ & 13,3 \\
\hline & \multicolumn{2}{|c|}{$\mathrm{CV}=16,9 \%$} & \multicolumn{2}{|c|}{$\mathrm{CV}=8,44 \%$} & \multicolumn{2}{|c|}{$\mathrm{CV}=13,0 \%$} \\
\hline \multirow[t]{2}{*}{7} & $31,6 \mathrm{Ba}$ & $39,2 \mathrm{Aba}$ & $35,0 \mathrm{Ba}$ & $34,5 \mathrm{BCa}$ & $11,0 \mathrm{~B}$ & 13,4 \\
\hline & \multicolumn{2}{|c|}{$\mathrm{CV}=28,2 \%$} & \multicolumn{2}{|c|}{$\mathrm{CV}=6,04 \%$} & \multicolumn{2}{|c|}{$\mathrm{CV}=26,7 \%$} \\
\hline \multirow[t]{2}{*}{14} & $31,0 \mathrm{Bb}$ & $33,2 \mathrm{Ba}$ & $39,8 \mathrm{Aa}$ & $36,6 \mathrm{~B}$ & $12,4 \mathrm{AB}$ & 12,2 \\
\hline & \multicolumn{2}{|c|}{$\mathrm{CV}=17,5 \%$} & \multicolumn{2}{|c|}{$\mathrm{CV}=7,81 \%$} & \multicolumn{2}{|c|}{$\mathrm{CV}=19,3 \%$} \\
\hline \multirow[t]{2}{*}{21} & $41,5 \mathrm{Aa}$ & 44,8 Aa & $32,8 \mathrm{Ba}$ & $32,5 \mathrm{C}$ & $13,6 \mathrm{AB}$ & 14,5 \\
\hline & \multicolumn{2}{|c|}{$\mathrm{CV}=14,6 \%$} & \multicolumn{2}{|c|}{$\mathrm{CV}=4,04 \%$} & \multicolumn{2}{|c|}{$\mathrm{CV}=12,6 \%$} \\
\hline \multirow[t]{2}{*}{28} & $43,1 \mathrm{Aa}$ & 44,4 Aa & $34,0 \mathrm{Ba}$ & $33,0 \mathrm{C}$ & $14,6 \mathrm{~A}$ & 14,6 \\
\hline & \multicolumn{2}{|c|}{$\mathrm{CV}=8,32 \%$} & \multicolumn{2}{|c|}{$\mathrm{CV}=5,00 \%$} & \multicolumn{2}{|c|}{$\mathrm{CV}=7,22 \%$} \\
\hline Referência $^{1}$ & \multicolumn{2}{|c|}{$31-48$} & \multicolumn{2}{|c|}{$32-36$} & \multicolumn{2}{|c|}{$10,0-14,2$} \\
\hline
\end{tabular}

ABC: médias seguidas das letras maiúsculas distintas apresentam diferença significante $(\mathrm{P}<0,05$, teste de Tukey) entre os tempos de coleta (coluna).

ab: médias seguidas das letras maiúsculas distintas apresentam diferença significante $(\mathrm{P}<0,05$, teste $\mathrm{t}$ de Student) entre os grupos (linhas)

${ }^{1}$ Segundo Clinical... (1988).

A CHGM representa a concentração da hemoglobina dentro de cada eritrócito. Conforme a concentração de hemoglobina, as anemias podem ser classificadas em hipocrômicas, quando essa concentração está reduzida, ou normocrômicas (Jain, 1993; Thrall et al., 2007). Ao se analisar o tempo zero (T0) de ambos os grupos, observam-se valores médios aumentados de CHGM (Tab. 2), que podem indicar um erro pré-analítico no hemograma automatizado. Algumas das causas de erro comuns na dosagem de CHGM são as crioaglutininas, hemólise in vitro, microcitose extrema ou coágulos (Dalanhol et al., 2010). De fato, muitas vezes não existe correlação perfeita do CHGM com a hipocromia no esfregaço de sangue. Apesar de se encontrar diminuída na anemia ferropriva, sua utilidade no diagnóstico desta condição é pequena, já que a queda de CHGM nestes casos ocorre mais tardiamente do que a queda de VGM (Jain, 1993).

A HGM reflete a massa total de hemoglobina no interior dos eritrócitos (Jain, 1993; Thrall et al., 2007). Os animais deste estudo apresentaram valores dentro dos limites de normalidade fisiológica (Tab. 2). Houve efeito do tempo do experimento nos valores de HGM nas éguas do grupo 1, com queda após sete dias de consumo do feno, mas com posterior recuperação; esses resultados refletem a concentração de hemoglobina, já relatada anteriormente.

Os valores médios de leucócitos totais (Tab. 3), nos dois grupos estudados, foram maiores na primeira coleta (T0) e menores na última coleta (T4), percebendo-se uma redução ao longo das coletas sanguíneas $(\mathrm{P}<0,05)$. Os valores mais 
altos observados na primeira coleta, possivelmente, foram decorrentes do estresse durante a coleta sanguínea (leucocitose fisiológica), com maior liberação de aminas biogênicas no sangue. Nas demais coletas, é provável que os animais tivessem menos estresse, indicando que tinham se acostumado com as coletas de sangue.

Tabela 3. Contagens médias e coeficiente de variação de leucócitos, linfócitos e neutrófilos de éguas alimentadas com feno (G1) ou haylage de Tifton-85 (G2)

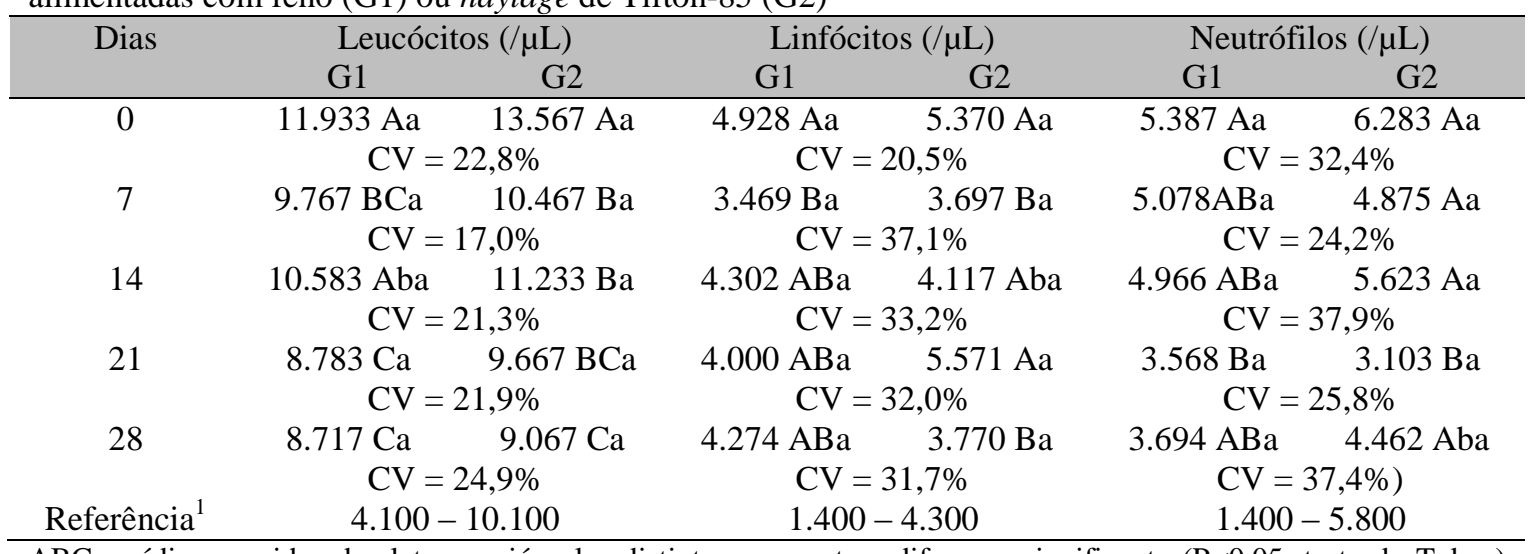

ABC: médias seguidas das letras maiúsculas distintas apresentam diferença significante ( $\mathrm{P}<0,05$, teste de Tukey) entre os tempos de coleta (coluna).

ab: médias seguidas das letras maiúsculas distintas apresentam diferença significante $(\mathrm{P}<0,05$, teste $t$ de Student) entre os grupos (linhas).

${ }^{1}$ Segundo Clinical... (1988).

Os valores de linfócitos dos animais do grupo 1 apresentaram linfocitose fisiológica na primeira coleta (Tab. 3). No grupo 2, ocorreu linfocitose em T1 e T3, coincidindo com os momentos de leucocitose fisiológica dos animais estudados. No entanto, em T5, os valores já se encontravam dentro da normalidade.

Nos neutrófilos (Tab. 3), não houve alteração fisiológica provocada pelos dois tipos de dietas fornecidas aos animais; somente foi observada uma diminuição $(\mathrm{P}<0,05)$ entre os tempos T0 e T3, em ambos os grupos. Em relação aos neutrófilos jovens, foram encontrados raros bastonetes em ambos os grupos submetidos às diferentes dietas e ao longo dos tempos de coleta, que são achados considerados fisiológicos (Thrall et al., 2007).

Os eosinófilos (Tab. 4), em T0, T1 e T2 em ambos os grupos, apresentaram valores acima do limite máximo de normalidade, todavia com diminuição gradativa até T4. A eosinofilia possui como causas mais comuns a infestação por diversos parasitas e as reações de hipersensibilidade (Thrall et al., 2007). Como esses valores estavam superiores aos de referência antes do fornecimento das dietas, é possível que as éguas não manifestassem alterações de hipersensibilidade à forrageira Tifton 85, tanto na forma de feno, quanto na forma de haylage.

As contagens de monócitos (Tab. 4) encontradas em ambos os grupos nos momentos avaliados estão acima dos limites de referência, exceto no grupo 1 em T4. Houve diminuição nas contagens dessas células com o tempo em ambos os grupos, mas sem significado clínico (Thrall et al., 2007). Do mesmo modo, apesar de ter havido alterações ao longo do tempo, os valores de basófilos (Tab. 4) se mantiveram dentro da normalidade fisiológica.

Quando bem produzidas, as haylages apresentam vantagem nutricional para os equinos devido à maior disponibilidade dos aminoácidos, que são absorvidos no intestino delgado antes de atingir o ceco-cólon (Muhonen et al., 2009). Essa melhora no aproveitamento da dieta torna a haylage o volumoso de eleição para integrar as dietas das categorias exigentes nesses nutrientes, como éguas no final de gestação e em lactação. Uma vantagem adicional é que, em decorrência das estações de monta, as éguas passam grande parte do final da gestação e, muitas vezes, da lactação 
no período de baixa pluviosidade, resultando em queda na qualidade nutricional, principalmente de proteínas (Costa, 2012). Essa indicação é reforçada pelos achados do presente trabalho, que não indicaram alterações hematológicas em decorrência da alimentação com haylage.

Tabela 4. Contagens médias e coeficiente de variação de eosinófilos, monócitos e basófilos de éguas alimentadas com feno (G1) ou haylage de Tifton-85 (G2)

\begin{tabular}{|c|c|c|c|c|c|c|}
\hline \multirow[t]{2}{*}{ Dias } & \multicolumn{2}{|c|}{ Eosinófilos $(/ \mu \mathrm{L})$} & \multicolumn{2}{|c|}{ Monócitos $(/ \mu \mathrm{L})$} & \multicolumn{2}{|c|}{ Basófilos $(/ \mu \mathrm{L})$} \\
\hline & G1 & $\mathrm{G} 2$ & G1 & $\mathrm{G} 2$ & G1 & $\mathrm{G} 2$ \\
\hline \multirow[t]{2}{*}{0} & 827 & 978 & $622 \mathrm{~A}$ & $838 \mathrm{~A}$ & $77,5 \mathrm{~B}$ & $42,8 \mathrm{C}$ \\
\hline & \multicolumn{2}{|c|}{$\mathrm{CV}=49,9 \%$} & \multicolumn{2}{|c|}{$\mathrm{CV}=63,3 \%$} & \multicolumn{2}{|c|}{$\mathrm{CV}=105,7 \%$} \\
\hline \multirow[t]{2}{*}{7} & 803 & 845 & $235 \mathrm{BCb}$ & $674 \mathrm{Aa}$ & $164 \mathrm{~A}$ & $171 \mathrm{AB}$ \\
\hline & \multicolumn{2}{|c|}{$\mathrm{CV}=52,7 \%$} & \multicolumn{2}{|c|}{$\mathrm{CV}=58,7 \%$} & \multicolumn{2}{|c|}{$\mathrm{CV}=98,3 \%$} \\
\hline \multirow[t]{2}{*}{14} & 595 & 938 & $403 \mathrm{AB}$ & $259 \mathrm{~B}$ & $48,0 \mathrm{C}$ & $206 \mathrm{~A}$ \\
\hline & \multicolumn{2}{|c|}{$\mathrm{CV}=60,7 \%$} & \multicolumn{2}{|c|}{$\mathrm{CV}=49,9 \%$} & \multicolumn{2}{|c|}{$\mathrm{CV}=119,5 \%$} \\
\hline \multirow[t]{2}{*}{21} & 526 & 344 & $491 \mathrm{AB}$ & $479 \mathrm{AB}$ & $154 \mathrm{~A}$ & $147 \mathrm{~B}$ \\
\hline & \multicolumn{2}{|c|}{$\mathrm{CV}=65,1 \%$} & \multicolumn{2}{|c|}{$\mathrm{CV}=51,5 \%$} & \multicolumn{2}{|c|}{$\mathrm{CV}=51,7 \%$} \\
\hline \multirow[t]{2}{*}{28} & 470 & 413 & $167 \mathrm{C}$ & $256 \mathrm{~B}$ & $111 \mathrm{AB}$ & $154 \mathrm{AB}$ \\
\hline & \multicolumn{2}{|c|}{$\mathrm{CV}=43,6 \%$} & \multicolumn{2}{|c|}{$\mathrm{CV}=59,2 \%$} & \multicolumn{2}{|c|}{$\mathrm{CV}=78,4 \%$} \\
\hline Referência $^{1}$ & \multicolumn{2}{|c|}{$0-500$} & \multicolumn{2}{|c|}{$0-200$} & \multicolumn{2}{|c|}{$0-300$} \\
\hline
\end{tabular}

AB: médias seguidas das letras maiúsculas distintas apresentam diferença significante $(\mathrm{P}<0,05$, teste de Tukey para eosinófilos e Friedman para monócitos e basófilos) entre os tempos de coleta (coluna).

ab: médias seguidas das letras maiúsculas distintas apresentam diferença significante $(\mathrm{P}<0,05$, teste $\mathrm{t}$ de Student para eosinófilos e Mann-Whitney para monócitos e basófilos) entre os grupos (linhas).

${ }^{1}$ Segundo Clinical... (1988).

\section{CONCLUSÃO}

A administração por 28 dias da forrageira Tifton85 (Cynodon spp.) na forma de pré-secado (haylage), com 70\% de MS para éguas Quarto de Milha, não causou alterações no perfil sanguíneo de equinos adultos.

\section{REFERÊNCIAS}

CLINICAL pathological services offered to the veterinary profession. Bedford: Beaufort Cottage Laboratories, [1988]. p.24.

COSTA, M.L.L. Utilização de haylage de tifton85 (Cynodon spp.) na dieta de eqüinos. 2012. 49f. Tese (Doutorado em Zootecnia). - Escola de Veterinária, Universidade Federal de Minas Gerais, Belo Horizonte, MG.
DALANHOL, M.; BARROS, M.; MAZUCHELLI, J. et al. Efeitos quantitativos da estocagem de sangue periférico na determinação de hemograma automatizado. Rev. Bras. Hematol. Hemoter., v.32, p.10-15, 2010.

DOMINGUES, J.L. Uso de volumosos conservados na alimentação de equinos. Rev. Bras. Zootec., v.38, Supl., p.259-269, 2009.

FORTES, E. Parasitologia Veterinária. 4a ed., São Paulo. Icone, 2004.

JAIN, N.C. Essentials of veterinary hematology. Philadelphia: Lea \& Febiger, 1993. 417p.

KUNG JR., L.; STOUGH, E.C.; McDONELL, E.E. et al. The effect of wide swathing on wilting times and nutritive value of alfafa haylage. $J$. Dairy Sci., v.93, p.1770-1773, 2010. 
MUHONEN, S.; JULLIAND, V.; LINDBERG, J.E. et al. Effects on the equine colon ecosystem of grass silage and haylage diets after an abrupt change from hay. J. Anim. Sci., v.87, p.22912298, 2009

MÜLLER, C.E.; UDÉN, P. Preference of horses for grass conserved as hay, haylage or silage. Anim. Feed Sci. Technol., v.132, p.66-78, 2007.

NUTRIENT requirements of horses. 6.ed. Washington: National Academy Press, 2007. 341p.

PASTORINI, L.H.; BACARIN, M.A.; ABREU, C.M. Secagem de material vegetal em forno de micro-ondas para determinação de matéria seca e análises químicas. Ciênc. Agrotéc., v.26, p.1252$1258,2002$.
RAGNARSSON, S.; LINDBERG, J.E. Nutritional value of timothy haylage in Icelandic horses. Livest. Sci., v.113, p.202-208, 2008.

RIET-CORREIA, F.; SCHILD, A.L.; MÉNDEZ, M.D. et al. Doenças de ruminantes e equinos. 2.ed. São Paulo: Varela, 2001. v.2, p.132-146.

SAMPAIO, I.B.M. Estatística aplicada a experimentação animal. 3.ed. Belo Horizonte: Fundação de Ensino e Pesquisa em Medicina Veterinária e Zootecnia, 2007. 264p.

THRALL, M.A.; CAMPBELL,T.; FETTMAN, M.J. et al. Hematologia e bioquímica clinica veterinária. São Paulo: Rocca, 2007. 582p.

VIEIRA, E.R., REZENDE, A.S.C.; LANA, A.M.Q. et al. Caracterização da equideocultura no estado de Minas Gerais. Arq. Bras. Med. Vet. Zootec., v.67, p.319-323, 2015. 